\title{
The upregulation of miR-204-3p in LPS-induced acute lung injury aggravated pulmonary endothelial cells apoptosis via targeting sulfatase 2
}

\author{
Liya Zhang ${ }^{1}$, Zhengyu Zhu², Qian Zhang ${ }^{\square}$ and Zhengdao Mao ${ }^{3}$

\begin{abstract}
'Department of Emergency Medicine, Nanjing Medical University Affiliated Changzhou No. 2 People's Hospital, Changzhou, Jiangsu Province, 213164, China; 2Department of Pediatrics, Nanjing Medical University Affiliated Changzhou No. 2 People's Hospital, Changzhou, Jiangsu Province, 213164, China; 'BDepartment of Respiratory Medicine, Nanjing Medical University Affiliated Changzhou No. 2 People's Hospital, Changzhou, Jiangsu Province, 213164, China
\end{abstract}

Acute lung injury (ALI) results from the injury of alveolar epithelial cells and pulmonary capillary endothelial cells, with a high mortality rate ranging from $29 \%$ to $42 \%$. Therefore, more efficient therapeutic strategies for ALI are necessary. Numerous studies revealed that miRNAs play a role in the regulation of ALI. Lipopolysaccharide (LPS) can induce an inflammatory response and has been widely applied in the establishment of the mouse ALI model. Here, we reported that miR-204-3p expression was upregulated by LPS treatment with increased cytokine secretion. LPS treatment promoted cell apoptosis, accompanied by abnormal cell structure and unobvious alveolar structure. These effects could be prevented by down-regulation of miR-204-3p, and promoted by miR-204-3p overexpression. Sulfatase 2 (SULF2) appeared to be the target of miR-204-3p predicted by TargetScan. Downregulation of miR-204-3p enhanced the protein level of SULF2, indicating that SULF2 was a target of miR-204-3p, which could negatively regulate the expression of SULF2. Thus, targeting miR-204-3p may be a potential therapeutic strategy for ALI.

Keywords: acute lung injury; sulfatase 2; miR-204-3p

Received: 15 October, 2020; revised: 26 November, 2020; accepted: 21 December, 2020; available on-line: 25 May, 2021

『e-mail: z qian1234@163.com

Abbreviations: ALI, acute lung injury; ARDS, acute respiratory distress syndrome; HSPGs, heparan sulfate proteoglycans. LPS, lipopolysaccharide; miRNAs, microRNAs; SULF2: sulfatase 2

\section{INTRODUCTION}

Acute lung injury (ALI) is the damage of alveolar epithelial cells and pulmonary capillary endothelial cells caused by non-cardiogenic factors. ALI is characterized by alveolar-capillary barrier damage, bilateral pulmonary infiltrates, non-cardiogenic pulmonary edema, poor lung compliance, and imbalance of ventilation/blood flow ratio (Ferguson et al., 2005; Levitt \& Matthay, 2006; Albus, 2012; Brown \& Watson, 2018). Patients with ALI often suffer from respiratory distress and refractory hypoxemia. There are various factors causing ALI, such as sepsis, pneumonia, aspiration of gastric contents, severe trauma, acute pancreatitis, and blood transfusion (Brown \& Watson, 2018). Indirect ALI is a common consequence of sepsis and about $40 \%$ of sepsis patients suffer from ALI (Brown \& Watson, 2018; Wang et al., 2019). Sepsis is caused by Gram-negative bacteria and lipopolysaccharide (LPS) plays a key role in the inflammatory response of
ALI (Brown \& Watson, 2018; Wang et al., 2019). Therefore, LPS can be used to establish the mouse ALI model. ALI may further develop into the severe stage named acute respiratory distress syndrome (ARDS) if not wellcontrolled (Ferguson et al., 2005; Levitt \& Matthay, 2006; Albus, 2012; Brown \& Watson, 2018). Although recently progress has been made in the diagnosis and treatment of ALI/ARDS, the mortality rate of ALI is still high, ranging from $29 \%$ to $42 \%$ depending on the therapeutic strategy and age (Ferguson et al., 2005; Levitt \& Matthay, 2006; Albus, 2012; Brown \& Watson, 2018). Therefore, it is urgent to find a more effective drug to control the development of ALI.

MicroRNAs (miRNAs) belong to non-coding RNAs (ncRNAs) that do not encode proteins and have a length of about $22 \mathrm{nt}$ (Sotiropoulou et al., 2009; Zhao \& Li, 2019). MiRNAs play an important role in the expression of $30 \%$ of protein-coding genes in vivo through interacting with the mRNA of the target gene at the 3'-untranslated region to initiate the inhibition of mRNA translation or induction of mRNA degradation (Sochor et al., 2014; Li et al., 2016). An increasing number of studies have reported the role of miRNAs in the regulation of ALI (Hara et al., 2007). For example, the inhibition of miRNA-494 alleviated ALI (Ling et al., 2018); upregulation of miR-146a suppressed the inflammatory responses in LPS-induced ALI (Zeng et al., 2013); miR-132 inhibited LPS-induced inflammation in alveolar macrophages (Liu et al., 2015); the inhibition of miR-34b-5p attenuated lung inflammation in ALI mouse model induced by LPS (Xie et al., 2018); downregulation of miR-181a attenuated LPS-induced ALI by targeting Bcl-2 ( $\mathrm{Li}$ et al., 2016). The accumulating evidence revealed that miRNAs exert an important role in the regulation of ALI.

In this study, we found that ALI could be alleviated by down-regulating miR-204-3p and aggravated by overexpressing miR-204-3p. Therefore, the inhibition of miR-204-3p may be a therapeutic strategy for ALI.

\section{MATERIALS AND METHODS}

\section{Cell lines and culture}

HEK293 and A549 cells lines were purchased from American Type Culture Collection (ATCC, USA). DMEM medium, F-12K medium, fetal bovine serum (FBS), Penicillin-Streptomycin, trypsin-EDTA were supplied by Thermo Fisher Scientific (China). HEK293 and A549 cells were cultured in DMEM and F-12K medium, 
respectively, supplemented with $10 \% \mathrm{FBS}, 100 \mathrm{IU} / \mathrm{ml}$ penicillin, $100 \mathrm{IU} / \mathrm{ml}$ streptomycin at $37^{\circ} \mathrm{C}$, in $5 \% \mathrm{CO}_{2}$ incubator.

\section{LPS-induced ALI mouse model}

Male C57BL6/J mice (8-10 weeks old) ( $\mathrm{n}=5$ per group) were anaesthetized and intratracheally instilled with $10 \mathrm{mg} / \mathrm{kg}$ LPS. Control mice were injected with equivalent pyrogen-free PBS. Saline was injected into the lung and immediately suctioned out to collect the alveolar lavage fluid. All animal experiments were approved by the Ethics Committee of Nanjing Medical University Affiliated Changzhou No.2 People's Hospital for the use of animals and conducted in accordance with the $\mathrm{Na}$ tional Institutes of Health Laboratory Animal Care and Use Guidelines.

\section{Quantitative reverse transcription polymerase chain reaction (qRT-PCR)}

Total RNA extraction was performed as previously described (Zhang et al., 2015; Caracciolo et al., 2019). Briefly, RNA was extracted from cells with Trizol (Invitrogen). qRT-PCR was performed using a SYBR PrimeScript RT-PCR kit (Takara Bio, USA) in ABI7900 system (Applied Biosystems, USA). U6 gene served as an internal control. The miR-204-3p transcript level was quantified using $2^{-\Delta \Delta \mathrm{Ct}}$ method (Schmittgen \& Livak, 2008).

\section{Enzyme-linked immunosorbent assay (ELISA)}

The levels of TNF- $\alpha$, IL- 6 and IL- $1 \beta$ in alveolar lavage fluid were assessed with ELISA kits purchased from Thermo Fisher Scientific. Each sample was measured in three technical replicates.

\section{miR-204-3p knockdown and overexpression}

To knockdown or overexpress miR-204-3p in vivo, male C57BL6/J mice (8-10 weeks old) ( $\mathrm{n}=5$ per group) were anaesthetized and intratracheally instilled with anti-miR-204-3p or miR-204-3p mimic, followed by LPS treatment after $24 \mathrm{~h}$. To knockdown or overexpress miR204-3p in vitro, miR-204-3p mimic or miR-204-3p inhibi- tor were transfected into cells using Lipofectamine ${ }^{\mathrm{TM}}$ 2000 supplied by Invitrogen (China).

\section{Western blotting}

The cell lysate was collected by centrifugation at $12000 \times g$ for $10 \mathrm{~min}$ after cells were lysed in radio-immunoprecipitation assay buffer (RIPA buffer) supplemented with protease inhibitor cocktail on ice for $30 \mathrm{~min}$. Bicinchoninic acid assay (BCA assay) was used to quantify the protein concentration in the supernatant. Proteins were separated by SDS-PAGE, then transferred onto PVDF membranes. After blocking with $5 \%(\mathrm{w} / \mathrm{v})$ dry non-fat milk and the incubation with primary antibodies, secondary antibodies were applied to the membranes. Blots were visualized with enhanced chemiluminescence. Relative protein expression was quantified with ImageJ software. $\beta$-actin served as the internal control. Primary antibodies against $\beta$-actin (ab8226), Bax (ab32503), Bcl-2 (ab32124), cleaved caspase-3 (ab2302), SULF2 (ab113405) and secondary antibody conjugated to horseradish peroxidase (HRP) were supplied by Abcam (China).

\section{Luciferase assay}

Cells were collected, rinsed twice with PBS, resuspended in Reporter Lysis Buffer (Promega Corporation, Madison, USA) at $1 \times 10^{6} / 100 \mu \mathrm{l}$, and incubated on ice for $20 \mathrm{~min}$. The supernatant was collected after the centrifugation at $12000 \times \mathrm{g}$ for $20 \mathrm{~min}$ and mixed with Luciferase Assay Reagent at the ratio of 1:5. The luminescence was detected with a luminometer.

\section{Hematoxylin-eosin (HE) staining}

Lung tissue was fixed for $24 \mathrm{~h}$ and dehydrated with xylene. The dehydrated tissue was embedded in paraffin, sliced, dried, and dewaxed. HE staining was performed using hematoxylin for $5 \mathrm{~min}$. After washing with distilled water, the color of hematoxylin was separated by alcohol hydrochloric acid (0.5-0.1\% hydrochloric acid diluted with $70 \%$ alcohol). Then the sections were stained with eosin for $2 \mathrm{~min}$, followed by dehydration, clearing, drying and mounting. An optical microscope was used to photograph the histological changes.

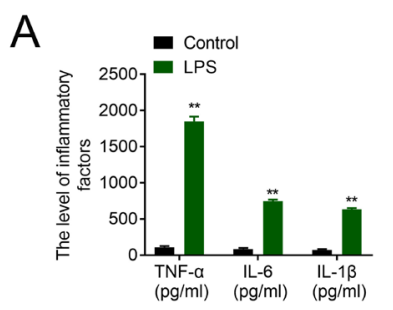

C

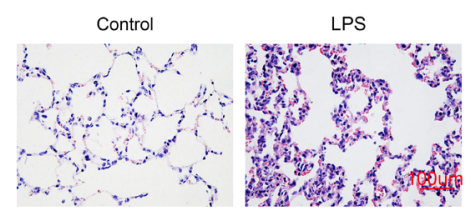

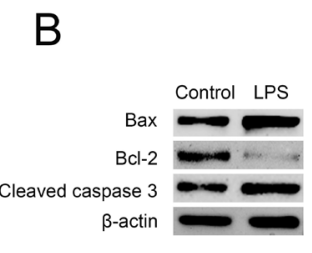

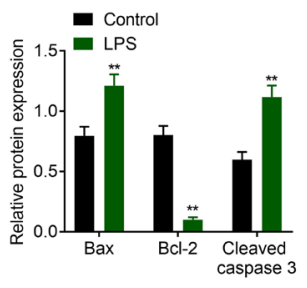

$\mathrm{D}$

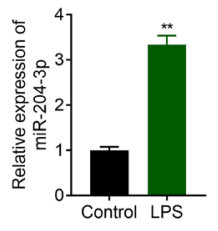

Figure 1. miR-204-3p was up-regulated in the ALI mouse model induced by LPS.

(A) ELISA was used to assess the levels of inflammatory cytokines in alveolar lavage fluid. ${ }^{* *} p<0.01$ vs control (ctrl). (B) Western blotting analysis was used to detect the expression levels of apoptosis-associated proteins, e.g. Bax, Bcl-2, cleaved caspase 3 . ${ }^{* *} p<0.01$ vs Control. (C) HE staining was used to observe the morphological structure change of lung tissue in LPS mice. (D) qPCR was used to measure the relative expression level of miR-204-3p. ${ }^{* *} p<0.01$ vs Control. Data are mean \pm S.E.M. of at least three independent experiments. 


\section{Cell viability}

A549 cells were transfected with miR-204-3p mimic or miR-204-3p inhibitor, then seeded at a concentration of $8 \times 10^{4}$ cells $/ \mathrm{ml}$ into a 96 -well plate $(100 \mu \mathrm{l}$ per well). After incubation for $24 \mathrm{~h}$, cells were treated with $1 \mu \mathrm{g} / \mathrm{ml}$ of LPS for another $24 \mathrm{~h}$. CCK-8 (Abcam, China) solution was added to the 96-well plate $\left(10 \mu \mathrm{l}\right.$ per well). The plate was incubated at $37^{\circ} \mathrm{C}$ for 1-4 hours. $A_{450 \mathrm{~nm}}$ was measured using a microplate reader.

\section{Statistical analysis}

Data are presented as mean \pm S.E.M. Statistical significance was analyzed using Student's $t$-test. $p<0.05$ was considered to be statistically significant.

\section{RESULTS}

\section{miR-204-3p was up-regulated in the ALI mouse model induced by LPS}

ALI mouse model was established by LPS intratracheal injection. ELISA assay revealed that secretion of TNF- $\alpha$, IL- 6 and IL-1 $\beta$ cytokines increased in the ALI mouse model (Fig. 1A). The protein expression of Bax and cleaved caspase 3 enhanced while the expression of Bcl-2 decreased after LPS treatment (Fig. 1B). $\mathrm{HE}$ staining demonstrated that normal lung tissue structure was clear and the alveolar space was large, but after LPS treatment, the lung tissue structure was abnormal and the alveolar structure was unobvious (Fig. 1C). The relative expression of miR-204-3p was increased by LPS treatment (Fig. 1D). These results suggested that LPS was able to induce lung injury and increase the expression level of miR-204-3p.

\section{Knockdown of miR-204-3p attenuated LPS-induced ALI}

Anti-miR-204-3p and miR-204-3p mimic were injected via tail vein. Similarly, LPS treatment increased the expression level of miR-204-3p (Fig. 2A). Injection of anti-miR-204-3p could reduce the level of miR-204-3p, and the injection of miR-204-3p mimic could further increase the level of miR-204-3p in LPS-induced ALI mice (Fig. 1A). LPS treatment significantly increased the levels of TNF- $\alpha$, IL- 6 and IL$1 \beta$ (Fig. 1B). The secretion of TNF- $\alpha$, IL- 6 and IL-1 $\beta$ was inhibited by reduced miR-204-3p level and promoted by increased miR-204-3p level (Fig. 2B). The protein expression of $\mathrm{Bax}$ and cleaved caspase 3 was reduced while $\mathrm{Bcl}-2$ expression was enhanced by antimiR-204-3p treatment in ALI mice (Fig. 1C). Overexpression of miR-204-3p resulted in the increase of Bax, the cleavage of caspase 3 and the decrease of Bcl-2 levels (Fig. 1C). The anti-miR-204-3p treatment alleviated alveolar damage in ALI mice, which, on the other hand, was enhanced by miR-204-3p overexpression (Fig. 1D). These results suggested that miR-204$3 \mathrm{p}$ downregulation attenuated LPS-induced ALI.

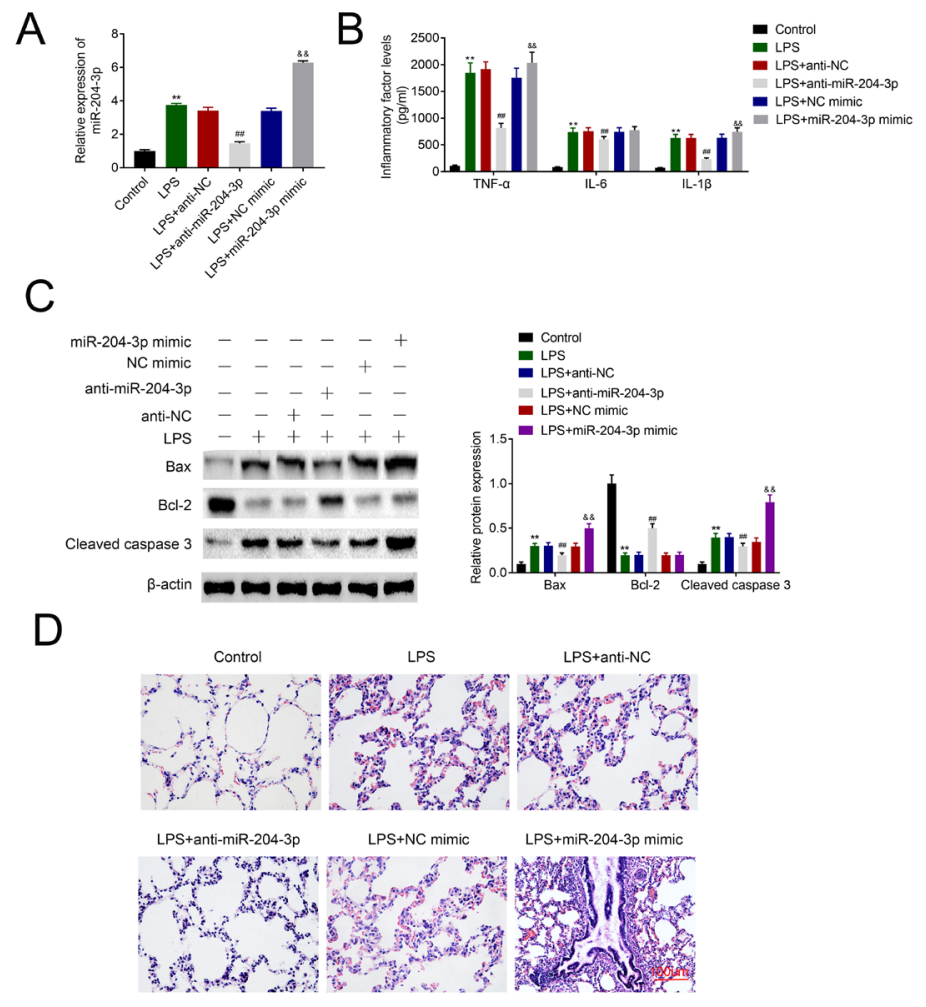

Figure 2. Knockdown of miR-204-3p attenuated LPS-induced ALI.

(A) qPCR was used to measure the relative expression level of miR-204-3p. ${ }^{* *} p<0.01$ vs Control, \#\# $p<0.01$ vs LPS+anti-NC, \&\& $p<0.01$ vs LPS+NC mimic. (B) ELISA was used to assess the levels of inflammatory cytokines in alveolar lavage fluid. ${ }^{* *} p<0.01$ vs Control, $\# \# p<0.01$ vs LPS+anti-NC, \&\&p $<0.01$ vs LPS+NC mimic. (C) Western blotting analysis was used to assess the expression levels of apoptosis-associated proteins, e.g. Bax, Bcl-2, cleaved caspase $3 .{ }^{* *} p<0.01$ vs Control, \#\# $p<0.01$ vs LPS+anti-NC, \&\& $p<0.01$ vs LPS+NC mimic. Data are mean \pm S.E.M. of at least three independent experiments. (D) HE staining was used to observe the morphological structure change of lung tissue in LPS mice. 


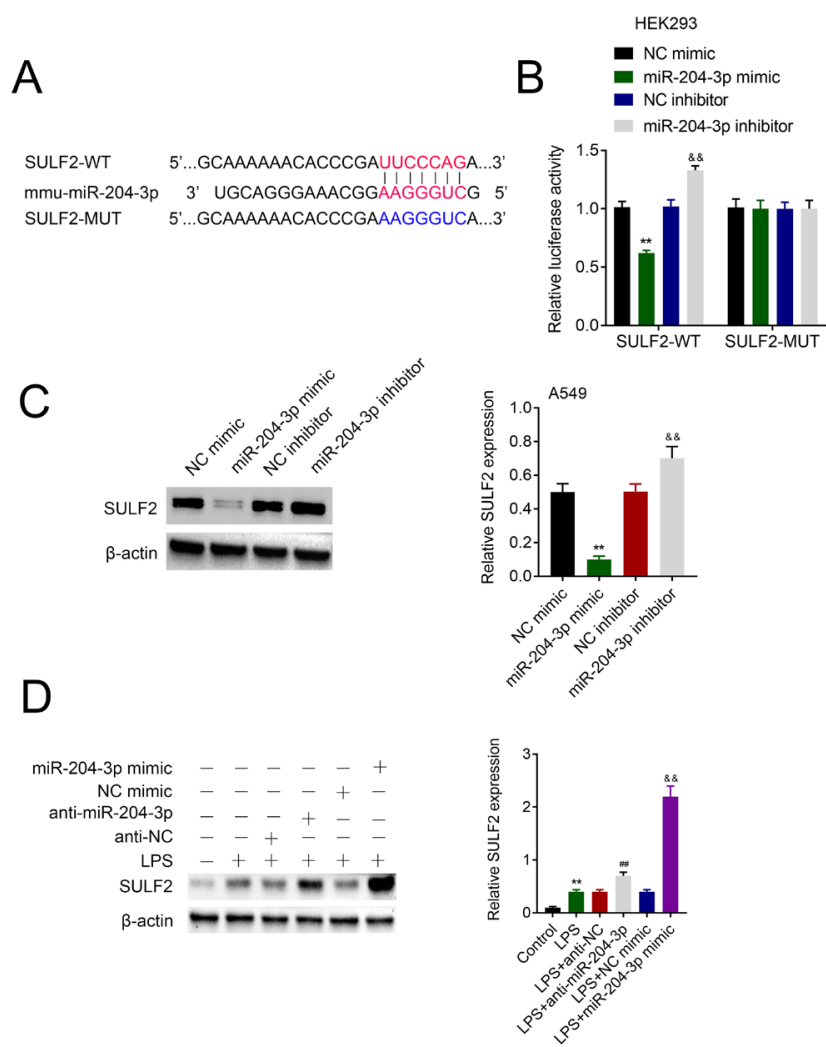

Figure 3. miR-204-3p targeted SULF2.

(A) TargetScan was performed to predict the possible target of miR-204-3p and to design the primers for the generation of SULF2 mutant. (B) Luciferase assay was used to test the effect of miR-204-3p mimic or miR-204-3p inhibitor treatment on the luciferase activity in SULF2 wild-type (SULF2-WT) and SULF2 mutant (SULF2-MUT) in HEK293 cells. ${ }^{* *} p<0.01$ vs NC mimic, \&\& $p<0.01$ vs NC inhibitor. (C) Western blotting was used to assess the protein level of SULF2 after treatment with miR-204-3p mimic or miR-204-3p inhibitor in A549 cells. Histograms represent the relative levels of SULF2 normalized to $\beta$-actin. ${ }^{* *} p<0.01$ vs NC mimic, ${ }^{8} \& p<0.01$ vs NC inhibitor. (D) Western blotting was used to assess the protein level of SULF2 after treatment with miR-204-3p mimic or miR-204-3p inhibitor in the lung tissue of LPSinduced ALI mice. Histograms represent the relative levels of SULF2 normalized to $\beta$-actin. ${ }^{* *} p<0.01$ vs Control, ${ }^{\# \#} p<0.01$ vs LPS+anti-NC, ${ }_{2} \& p<0.01$ vs LPS+NC mimic. Data are mean \pm S.E.M. of at least three independent experiments.

A
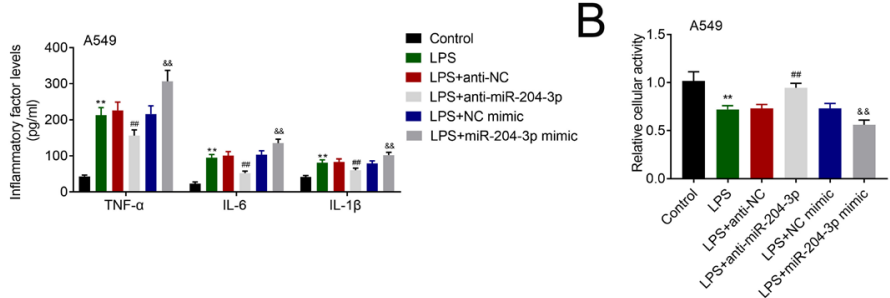

C
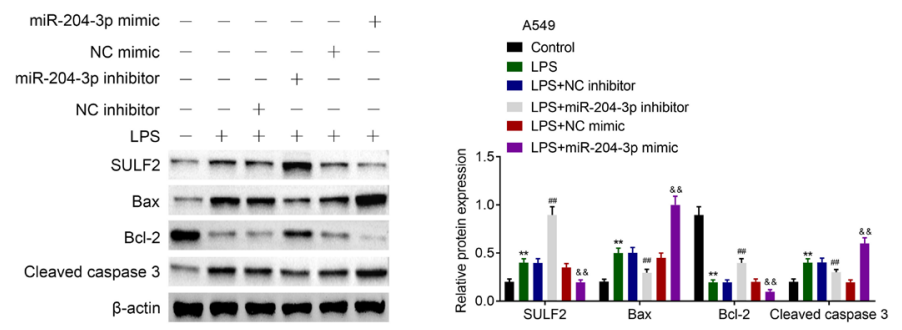

Figure 4. miR-204-3p regulated LPS-induced cell apoptosis via SULF2.

(A) ELISA was used to measure the levels of inflammatory cytokines in LPS-treated A549 cells. ${ }^{* *} p<0.01$ vs Control, ${ }^{* \#} p<0.01$ vs LPS+antiNC, \&\&p $<0.01$ vs LPS+NC mimic. (B) CCK-8 was used to assess the effect of LPS treatment and miR-204-3p overexpression on the viability of A549 cells. ${ }^{* *} p<0.01$ vs Control, $\#$ \#\# $<0.01$ vs LPS+anti-NC, \&\&p $<0.01$ vs LPS+NC mimic. (C) Western blotting analysis was used to assess the expression levels of SULF2 and apoptosis-associated proteins, e.g. Bax, Bcl-2, cleaved caspase $3 .{ }^{* *} p<0.01$ vs Control, ${ }^{* \#} p<0.01$ vs LPS + NC inhibitor, ${ }^{2} \& p<0.01$ vs LPS+NC mimic. Data are mean \pm S.E.M. of at least three independent experiments. 


\section{miR-204-3p targeted SULF2}

TargetScan (http://www.targetscan.org/) was performed to predict the possible target of miR-204-3p (Fig. 3A). In SULF2 wild-type (WT) HEK293 cells, miR-204-3p overexpression decreased the relative activity of luciferase, while the treatment with miR-204$3 p$ inhibitor increased the relative activity of luciferase, which could not be detected in the case of SULF2 mutant (SULF2-MUT) (Fig. 3B). Luciferase assay revealed that miR-204-3p interacted with SULF2, and the effect of miR-204-3p on the expression of SULF2 was detected. In human alveolar basal epithelial A549 cells, overexpression of miR-204-3p inhibited the expression of SULF2, while miR-204-3p inhibitor increased the expression of SULF2 (Fig. 3C). In LPS-induced ALI mice, the expression of SULF2 was increased (Fig. 3D). To further confirm the interaction between miR-204-3p and SULF2, the expression of SULF2 in LPS-induced ALI mice was assessed after miR-204-3p knockdown or overexpression. The treatment with anti-miR-204-3p further increased the protein level of SULF2 (Fig. 3D). These results revealed that miR-204-3p targeted and negatively regulated the expression of SULF2.

\section{miR-204-3p regulated LPS-induced cell apoptosis via SULF2}

To verify the interaction of miR-204-3p and SULF2, and its effect on LPS-induced cell death, A549 cells were used for the corresponding tests. In LPS-treated A549 cells, the secretion of TNF- $\alpha$, IL- 6 and IL- $1 \beta$ cytokine was increased, and the effect was reduced by anti-miR204-3p treatment and promoted by miR-204-3p mimic (Fig. 4A). LPS treatment induced cell death, which was prevented by the inhibitor of miR-204-3p and aggravated by miR-204-3p overexpression (Fig. 4B). In LPS-treated A549 cells, the treatment with miR-204-3p inhibitor elevated the expression of SULF2 and Bcl-2, which was prevented by miR-204-3p overexpression (Fig. 4C). These results suggested that miR-204-3p regulated LPSinduced cell apoptosis via SULF2.

\section{DISCUSSION}

ALI is the damage of alveolar epithelial cells and pulmonary capillary endothelial cells (Ferguson et al., 2005; Levitt \& Matthay, 2006; Albus, 2012; Brown \& Watson, 2018). It can be caused by various factors, such as sepsis, pneumonia, aspiration of stomach contents, acute pancreatitis, and blood transfusion (Brown \& Watson 2018). ALI can progress into ARDS which is characterized by a high mortality rate (Ferguson et al., 2005; Levitt \& Matthay, 2006; Albus, 2012; Brown \& Watson, 2018). Increasing evidence demonstrated that microRNAs control the expression of many protein-coding genes and play an important role in the regulation of various diseases including ALI (Hara et al., 2007; Sochor et al., 2014; Li et al., 2016).

By removing 6-O-sulfates from specific heparan sulfate intrachain sites, SULF2 regulates the functions of many growth factors and cytokines (Yue, 2017). SULF2 was proved to be involved in many signaling pathways by mobilizing protein ligands from the sequestration by heparan sulfate proteoglycans (HSPGs) thus liberating the ligands for binding to signal transduction receptors, and SULF2 was also identified as a driver of carcinogenesis (Lui et al., 2016; Li et al., 2019). SULF2 was overexpressed in the idiopathic pulmonary fibrosis lungs.
Knockdown of SULF2 aggravated lung injury induced by bleomycin, demonstrating that SULF2 protected hyperplastic type II alveolar epithelial cells from epithelial injury, inflammation, and cell death (Yue, 2017). However, the protective mechanism of SULF2 on pulmonary endothelial cells remains to be further investigated. We found that the expression of miR-204-3p was upregulated in LPS-induced ALI and miR-204-3p targeted and inhibited the expression of SULF2. MicroRNAs inhibit cancer development by targeting SULF2 (Ling et al., 2018; Li et al., 2019). In this work, the role of microRNA in the regulation of ALI and the possible involvement of SULF2 were investigated.

LPS was used to induce an inflammatory response to establish the ALI mouse model. In this study, the expression of miR-204-3p was upregulated in LPS-induced ALI mouse model, indicating that the expression level of miR-204-3p may be used as a biomarker for ALI diagnosis. Correspondingly, the secretion of TNF- $\alpha$, IL- 6 and IL-1 $\beta$ cytokines was increased in the ALI mice model, suggesting that the inflammatory response was induced by LPS treatment. The protein expression level of Bax and cleaved caspase 3 increased while the expression of $\mathrm{Bcl}-2$ decreased after LPS treatment, suggesting that LPS treatment induced cell apoptosis. To further investigate the role of miR-204-3p in ALI, the expression of miR204-3p was downregulated or upregulated in vivo and in vitro. The cytokine secretion was inhibited by downregulation of miR-204-3p and promoted by miR-204-3p overexpression. Moreover, lung cell apoptosis was prevented by miR-204-3p inhibition and promoted by miR204-3p overexpression. The lung tissue in LPS-treated mice exhibited abnormal structure and unobvious alveolar structure, which was alleviated by downregulation of miR-204-3p and deteriorated by miR-204-3p overexpression. These results demonstrated that miR-204-3p aggravated the development of ALI and targeting miR-204$3 p$ may be a therapeutic strategy for ALI treatment. The potential regulatory mechanism of miR-204-3p in ALI was further explored. Luciferase assay suggested that miR-204-3p targeted and negatively regulated the expression of SULF2, indicating that overexpression of miR204-3p promoted ALI via inactivation of the expression of SULF2.

In the present study, we verified that miR-204-3p was upregulated in ALI and miR-204-3p could target and inhibit the expression of SULF2. This finding provided a molecular target for the treatment of ALI.

\section{Acknowledgements}

Not applicable.

\section{Funding}

Not applicable.

\section{Competing interests}

The authors state that there are no conflicts of interest to disclose.

\section{Ethics approval}

All animal experiments were approved by the Ethics Committee of Nanjing Medical University Affiliated Changzhou No.2 People's Hospital for the use of animals and conducted in accordance with the National Institute of Health Laboratory Animal Care and Use Guidelines. 


\section{Availability of data and materials}

All data generated or analyzed during this study are included in this published article.

\section{Authors' contributions}

Liya Zhang and Zhengyu Zhu designed the study, supervised the data collection, Zhengdao Mao analyzed the data, interpreted the data, Qian Zhang prepared the manuscript for publication and reviewed the draft of the manuscript. All authors have read and approved the manuscript.

\section{REFERENCES}

Albus U (2012) Guide for the Care and Use of Laboratory Animals (8th edn), SAGE Publications Sage UK: London, England

Brown AS, Watson KE (2018) Statin Intolerance. Reviews in Cardiovascular Medicine 19: 9-19.

Caracciolo D, Di Martino MT, Amodio N, Morelli E, Montesano M, Botta C, Scionti F, Talarico D, Altomare E, Gallo Cantafio ME, Zuccalà V, Maltese L, Todoerti K, Rossi M, Arbitrio M, Neri A, Tagliaferri P, Tassone P (2019) miR-22 suppresses DNA ligase III addiction in multiple myeloma. Leukemia 33: 487-498. https://doi. org/10.1038/s41375-018-0238-2

Ferguson ND, Frutos-Vivar F, Esteban A, Fernández-Segoviano P, Aramburu JA, Nájera L, Stewart TE (2005) Acute respiratory distress syndrome: underrecognition by clinicians and diagnostic accuracy of three clinical definitions. Crit Care Med 33: 2228-2234. https://doi. org/10.1097/01.ccm.0000181529.08630.49

Hara S, Mukai T, Kurosaki K, Mizukami H, Kuriiwa F, Endo T (2007) Role of nitric oxide system in hydroxyl radical generation in rat striatum due to carbon monoxide poisoning, as determined by microdialysis. Toxicology 239: 136-143. https://doi.org/10.1016/j. tox.2007.07.008

Levitt JE, Matthay MA (2006) Treatment of acute lung injury: historical perspective and potential future therapies. Semin Respir Crit Care Med 27: 426-437. https://doi.org/10.1055/s-2006-948296

Li Q, Li X, Wang L, Zhang Y, Chen L (2016) miR-98-5p acts as a target for Alzheimer's disease by regulating A $\beta$ production through modulating SNX6 expression. J Mol Neurosci 60: 413-420. https:// doi.org/10.1007/s12031-016-0815-7

Li W, Qiu X, Jiang H, Han Y, Wei D, Liu J (2016) Downregulation of miR-181a protects mice from LPS-induced acute lung injury by targeting Bcl-2. Biomed Pharmacother 84: 1375-1382. https://doi. org/10.1016/j.biopha.2016.10.065

Li WQ, Zhang JP, Wang YY, Li XZ, Sun L (2019) MicroRNA-422a functions as a tumor suppressor in non-small cell lung cancer through SULF2-mediated TGF-beta/SMAD signaling pathway. Cell
Cycle 18: 1727-1744. https://doi.org/10.1080/15384101.2019.16321 35

Ling Y, Li ZZ, Zhang JF, Zheng XW, Lei ZQ, Chen RY, Feng JH (2018) MicroRNA-494 inhibition alleviates acute lung injury through Nrf2 signaling pathway via NQO1 in sepsis-associated acute respiratory distress syndrome. Life Sci 210: 1-8. https://doi.org/10.1016/j. lfs.2018.08.037

Liu F, Li Y, Jiang R, Nie C, Zeng Z, Zhao N, Huang C, Shao Q, Ding C, Qing C, Xia L, Zeng E, Qian K (2015) miR-132 inhibits lipopolysaccharide-induced inflammation in alveolar macrophages by the cholinergic anti-inflammatory pathway. Exp Lung Res 41: 261-269. https://doi.org/10.3109/01902148.2015.1004206

Lui NS, Yang YW, van Zante A, Buchanan P, Jablons DM, Lemjabbar-Alaoui H (2016) SULF2 Expression Is a Potential Diagnostic and Prognostic Marker in Lung Cancer. PLoS One 11: e0148911. https://doi.org/10.1371/journal.pone.0148911

Schmittgen TD, Livak KJ (2008) Analyzing real-time PCR data by the comparative CT method. Nat Prot 3: 1101-1108. https://doi. org/10.1038/nprot.2008.73

Sochor M, Basova P, Pesta M, Dusilkova N, Bartos J, Burda P, Pospisil V, Stopka T (2014) Oncogenic MicroRNAs: miR-155, miR19a, miR-181b, and miR-24 enable monitoring of early breast cancer in serum. BMC Cancer 14: 448. https://doi.org/10.1186/14712407-14-448

Sotiropoulou G, Pampalakis G, Lianidou E, Mourelatos Z (2009) Emerging roles of microRNAs as molecular switches in the integrated circuit of the cancer cell. RNA 15: 1443-1461. https://doi. org/10.1261/rna.1534709

Wang L, Xu J, Guo D, Zhou X, Jiang W, Wang J, Tang J, Zou Y, Bi M, Li Q (2019) Fasudil alleviates brain damage in rats after carbon monoxide poisoning through regulating neurite outgrowth inhibitor/oligodendrocytemyelin glycoprotein signalling pathway. Basic Clin Pharmacol Toxicol 125: 152-165. https://doi.org/10.1111/ bcpt. 13233

Xie W, Lu Q, Wang K, Lu J, Gu X, Zhu D, Liu F, Guo Z (2018) miR-34b-5p inhibition attenuates lung inflammation and apoptosis in an LPS-induced acute lung injury mouse model by targeting progranulin. J Cell Physiol 233: 6615-6631. https://doi.org/10.1002/ jcp. 26274

Yue X (2017) Epithelial deletion of Sulf2 exacerbates bleomycin-induced lung injury, inflammation, and mortality. Am J Respir Cell Mol Biol 57: 560-569. https://doi.org/10.1165/rcmb.2016-0367OC

Zeng Z, Gong H, Li Y, Jie K, Ding C, Shao Q, Liu F, Zhan Y, Nie C, Zhu W, Qian K (2013) Upregulation of miR-146a contributes to the suppression of inflammatory responses in LPS-induced acute lung injury. Exp Lung Res 39: 275-282. https://doi.org/10.3109/01 902148.2013.808285

Zhang H, Tang J, Li C, Kong J, Wang J, Wu Y, Xu E, Lai M (2015) MiR-22 regulates 5 -FU sensitivity by inhibiting autophagy and promoting apoptosis in colorectal cancer cells. Cancer Lett 356: 781790. https://doi.org/10.1016/j.canlet.2014.10.029

Zhao W, Li L (2019) SP1-induced upregulation of long non-coding RNA HCP5 promotes the development of osteosarcoma. Pathol Res Pract 215: 439-445. https://doi.org/10.1016/j.prp.2018.12.006 